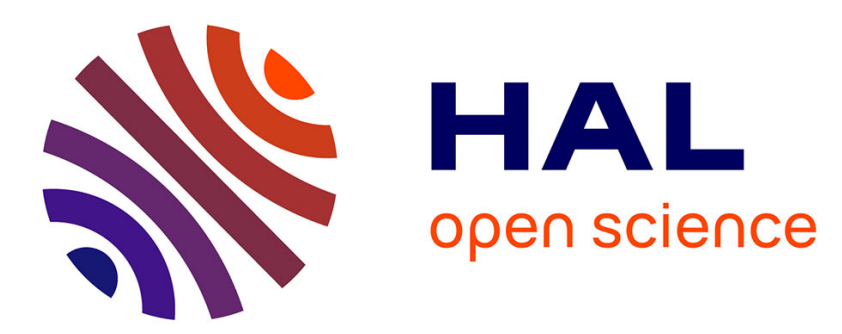

\title{
Efficient Leak Resistant Modular Exponentiation in RNS
}

Andrea Lesavourey, Christophe Negre, Thomas Plantard

\section{To cite this version:}

Andrea Lesavourey, Christophe Negre, Thomas Plantard. Efficient Leak Resistant Modular Exponentiation in RNS. ARITH: Computer Arithmetic, Jul 2017, London, United Kingdom. pp.156-163, 10.1109/ARITH.2017.39 . lirmm-01925642

\section{HAL Id: lirmm-01925642 \\ https://hal-lirmm.ccsd.cnrs.fr/lirmm-01925642}

Submitted on 16 Nov 2018

HAL is a multi-disciplinary open access archive for the deposit and dissemination of scientific research documents, whether they are published or not. The documents may come from teaching and research institutions in France or abroad, or from public or private research centers.
L'archive ouverte pluridisciplinaire HAL, est destinée au dépôt et à la diffusion de documents scientifiques de niveau recherche, publiés ou non, émanant des établissements d'enseignement et de recherche français ou étrangers, des laboratoires publics ou privés. 


\section{Efficient Leak Resistant Modular Exponentiation in RNS}

Andrea Lesavourey ${ }^{(1)}$, Christophe Negre $^{(1)}$ and Thomas Plantard ${ }^{(2)}$

(1) DALI (UPVD) and LIRMM (Univ. of Montpellier, CNRS), Perpignan, France

(2) CCISR, SCIT, University of Wollongong, Wollongong, Australia

24-th Symposium on Computer Arithmetic,

London, July 26, 2017
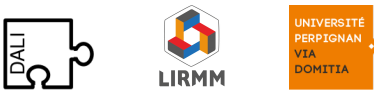


\section{Outline}

(1) Cryptography

- RSA cryptosystem

- Power analysis

- Montgomery multiplication in RNS

(2) Randomized modular exponentiation in RNS

- Randomized Montgomery multiplication

- Proposed approach

- Level of randomization

(3) Conclusion 


\section{Outline}

(1) Cryptography

- RSA cryptosystem

- Power analysis

- Montgomery multiplication in RNS

Randomized modular exponentiation in RNS

- Randomized Montgomery multiplication

- Proposed approach

- Level of randomization 


\section{RSA encryption (Rivest, Shamir and Adleman)}

Bob chooses $p$ and $q$ two large prime numbers and computes $N=p q$. He generates $E$ and $D$ two integers such that $E D=1(\bmod (p-1)(q-1))$.

- Public Key: N, D.

- Private Key: $E, p, q$.

- Alice encrypts a message $m$ by: $c=m^{D} \bmod N$.

- Bob decrypts $c$ by doing: $c^{E}=m^{E D} \bmod N=m$. 
An algorithm for modular exponentiation : Right-to-left Square-and-multiply

Require: A modulus $N$, an

integer $X \in[0, N[$ and an

exponent

$$
E=\left(e_{\ell-1}, \ldots, e_{0}\right)_{2}
$$

Ensure: $R=X^{E}(\bmod N)$

1: $R \leftarrow 1$

2: $Z \leftarrow X$

$$
X^{E}=X^{\sum_{i=0}^{\ell-1} e_{i} 2^{i}}
$$

3: for $i$ from 0 to $\ell-1$ do

4: $\quad$ if $e_{i}=1$ then

5: $\quad R \leftarrow R \times Z(\bmod N)$

$$
X^{E}=X^{e_{\ell-1} 2^{\ell-1}} \times \cdots \times X^{e_{1} 2^{1}} \times X^{e_{0} 2^{0}}
$$

6: end if

7: $\quad Z \leftarrow Z^{2}(\bmod N)$

8: end for

9: return $R$ 


\section{Simple power analysis}

$E=\left(e_{\ell}, \ldots, e_{0}\right)_{2}$ and $X \in[0, N[$

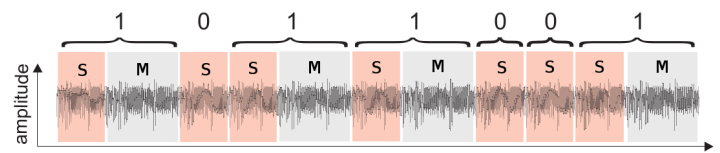

$\uparrow$

\begin{tabular}{|l|}
\hline Square-and-multiply \\
\hline$R \leftarrow 1$ \\
$Z \leftarrow X$ \\
for $i=0$ to $\ell-1$ do \\
if $e_{i}=1$ then \\
$\quad R \leftarrow R \cdot Z \bmod N$ \\
endif \\
$Z \leftarrow Z^{2} \bmod N$ \\
endfor \\
return $(R)$ \\
\end{tabular}




\section{Simple power analysis}

$$
E=\left(e_{\ell}, \ldots, e_{0}\right)_{2} \text { and } X \in[0, N[
$$

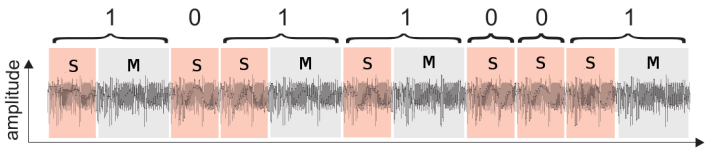

\begin{tabular}{|l|} 
个quare-and-multiply \\
\hline$R \leftarrow 1$ \\
$Z \leftarrow X$ \\
for $i=0$ to $\ell-1$ do \\
if $e_{i}=1$ then \\
$R \leftarrow R \cdot Z \bmod N$ \\
endif $\quad$ mod $N$ \\
$Z \leftarrow Z^{2} \operatorname{mor}$ \\
endfor \\
return $(R)$
\end{tabular}
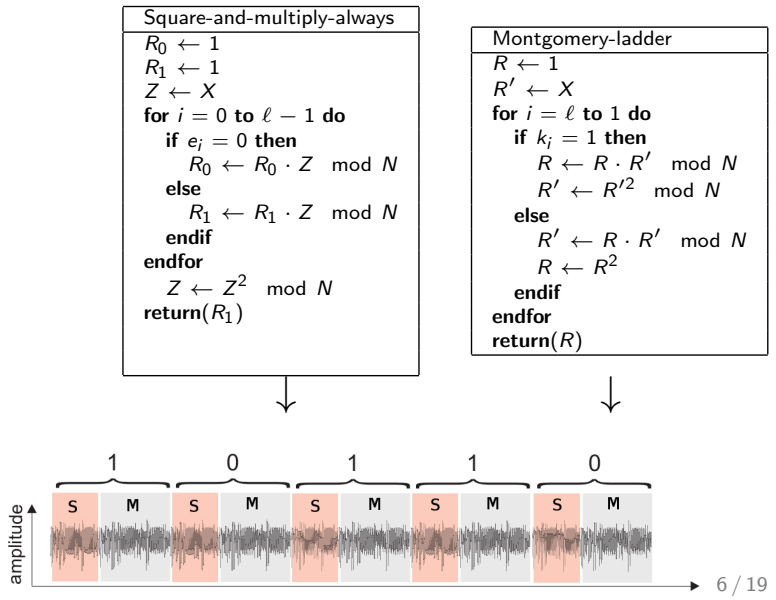


\section{Differential power analysis}

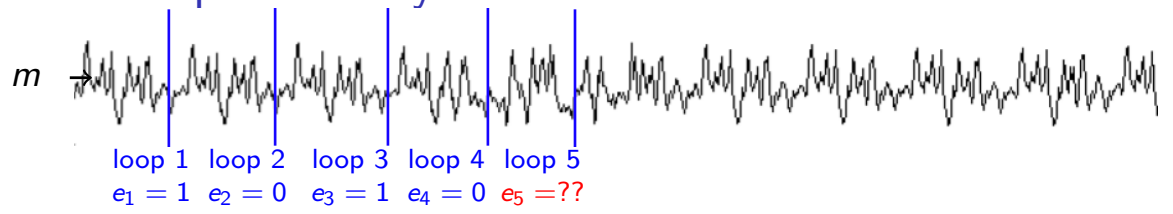




\section{Differential power analysis}

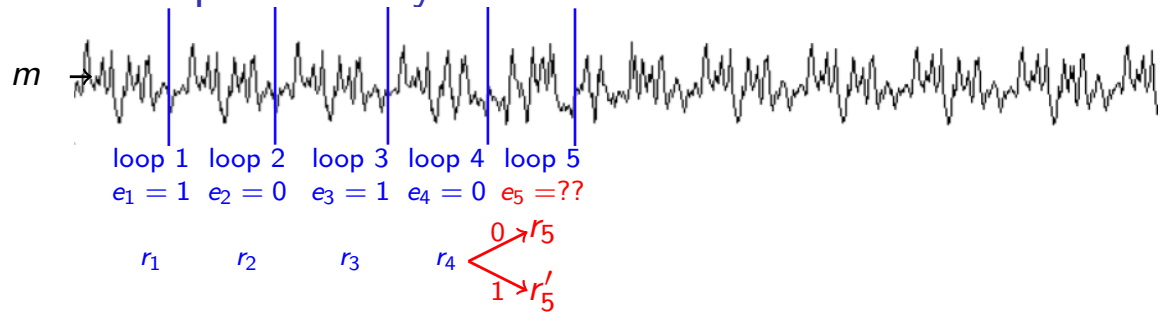




\section{Differential power analysis}

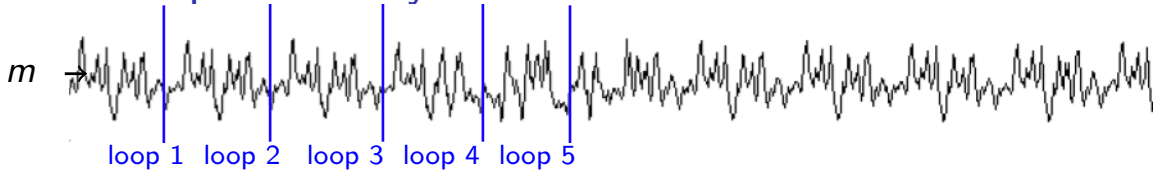

$$
\begin{aligned}
& e_{1}=1 \quad e_{2}=0 \quad e_{3}=1 \quad e_{4}=0 \quad e_{5}=\text { ?? } \\
& \begin{array}{lll}
r_{1} & r_{2} & r_{3}
\end{array} \\
& r_{4} \sum_{1>r_{5}^{\prime}}^{0>r_{5}}
\end{aligned}
$$

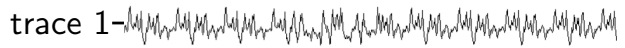

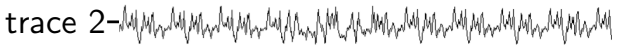

trace 3-

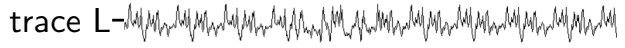




\section{Differential power analysis}

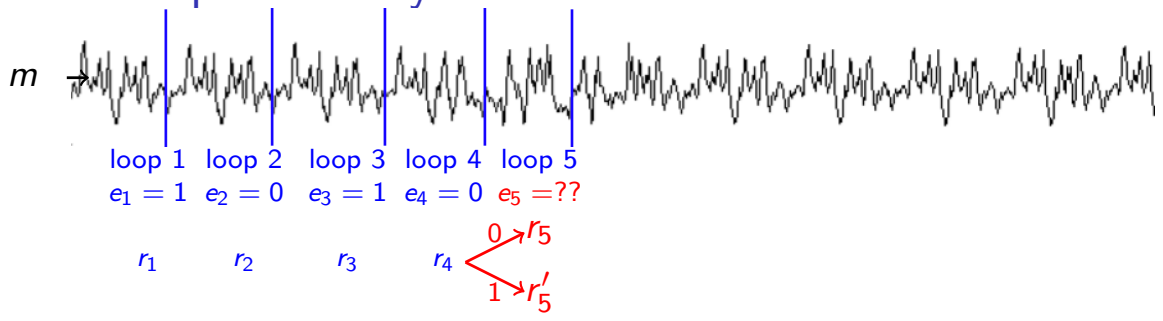

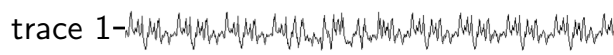

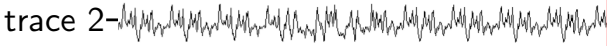
trace 3Differentials:

\section{correct guess}

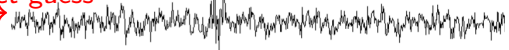

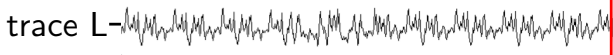




\section{Differential power analysis}

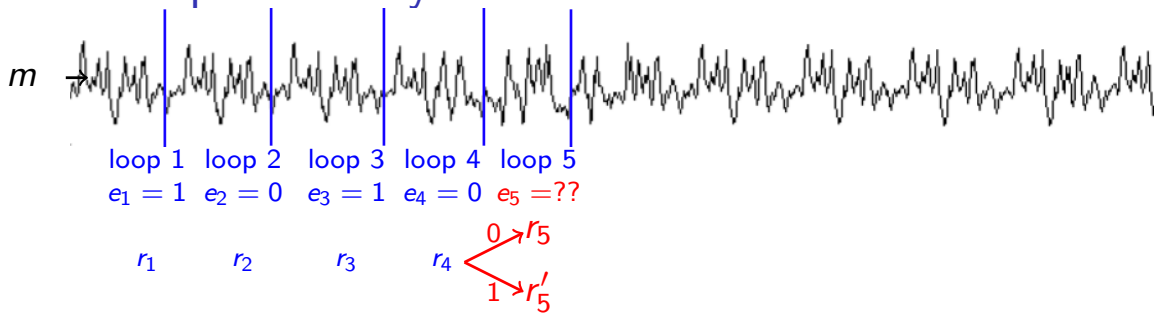

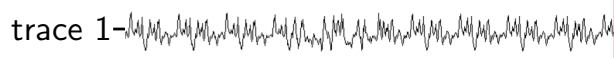

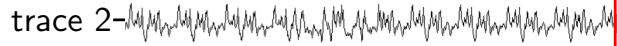
trace 3-

Differentials:

\section{correct guess}

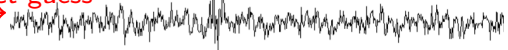

\section{wrong guess}

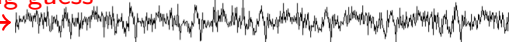

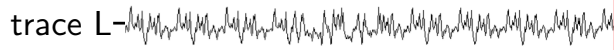

Counter-measure: Randomization of the exponent and data. 


\section{Montgomery multiplication}

Basic modular multiplication. For $X, Y \in[0, N[$

(1) Product. $Z \leftarrow X \times Y$

(2) Reduction. $Q \leftarrow\lfloor Z / N\rfloor$ and $R \leftarrow Z-Q \times N$ 


\section{Montgomery multiplication}

Basic modular multiplication. For $X, Y \in[0, N[$

(1) Product. $Z \leftarrow X \times Y$

(2) Reduction. $Q \leftarrow\lfloor Z / N\rfloor$ and $R \leftarrow Z-Q \times N$

\section{Montgomery Multiplication}

Require: $X, Y \in[0, N[$ and

$$
A=2^{n}>N
$$

Ensure: $R=X \times Y \times A^{-1}(\bmod N)$

1: $Z \leftarrow X \times Y$

2: $Q \leftarrow N^{-1} \times Z(\bmod A)$

3: $R \leftarrow(Z-Q \times N) / A$

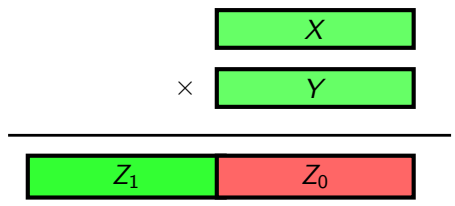




\section{Montgomery multiplication}

Basic modular multiplication. For $X, Y \in[0, N[$

(1) Product. $Z \leftarrow X \times Y$

(2) Reduction. $Q \leftarrow\lfloor Z / N\rfloor$ and $R \leftarrow Z-Q \times N$

\section{Montgomery Multiplication}

Require: $X, Y \in[0, N[$ and

$$
A=2^{n}>N
$$

Ensure: $R=X \times Y \times A^{-1}(\bmod N)$

$$
\text { 1: } Z \leftarrow X \times Y
$$

2: $Q \leftarrow N^{-1} \times Z(\bmod A)$

3: $R \leftarrow(Z-Q \times N) / A$

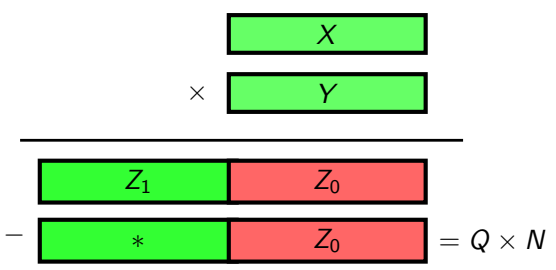




\section{Montgomery multiplication}

Basic modular multiplication. For $X, Y \in[0, N[$

(1) Product. $Z \leftarrow X \times Y$

(2) Reduction. $Q \leftarrow\lfloor Z / N\rfloor$ and $R \leftarrow Z-Q \times N$

\section{Montgomery Multiplication}

Require: $X, Y \in[0, N[$ and

$$
A=2^{n}>N
$$

Ensure: $R=X \times Y \times A^{-1}(\bmod N)$

1: $Z \leftarrow X \times Y$

2: $Q \leftarrow N^{-1} \times Z(\bmod A)$

3: $R \leftarrow(Z-Q \times N) / A$

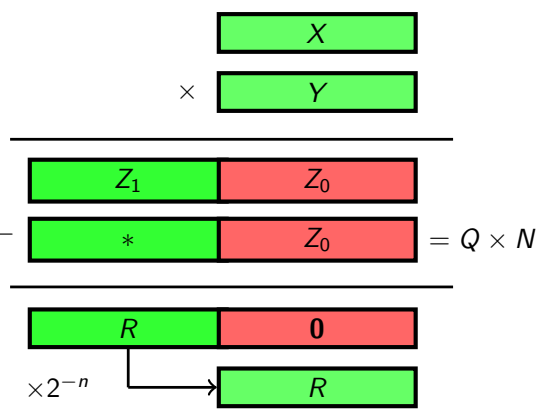




\section{Montgomery multiplication}

Basic modular multiplication. For $X, Y \in[0, N[$

(1) Product. $Z \leftarrow X \times Y$

(2) Reduction. $Q \leftarrow\lfloor Z / N\rfloor$ and $R \leftarrow Z-Q \times N$

\section{Montgomery Multiplication}

Require: $X, Y \in[0, N[$ and

$$
A=2^{n}>N
$$

Ensure: $R=X \times Y \times A^{-1}(\bmod N)$

$$
\text { 1: } Z \leftarrow X \times Y
$$$$
\text { 2: } Q \leftarrow N^{-1} \times Z(\bmod A)
$$$$
\text { 3: } R \leftarrow(Z-Q \times N) / A
$$

Montgomery representation.

(1) $\widetilde{X}=X A \bmod N$ provides

(2) $\operatorname{MontMul}(\tilde{X}, \widetilde{Y})=(X A) \times(Y A) \times A^{-1} \bmod N=X Y A \bmod N$ 
Montgomery multiplication in residue number system

- Let $\mathcal{A}=\left\{a_{1}, \ldots, a_{t}\right\}$ be a set $t$ co-prime integers. 


\section{Montgomery multiplication in residue number system}

- Let $\mathcal{A}=\left\{a_{1}, \ldots, a_{t}\right\}$ be a set $t$ co-prime integers.

- An integer $X$ such that $0 \leq X<A=\prod_{i=1}^{t} a_{i}$ is represented by

$$
[X]_{\mathcal{A}}=\left(x_{1}=X \quad \bmod a_{1}, \ldots, x_{t}=X \quad \bmod a_{t}\right) .
$$


Montgomery multiplication in residue number system

- Let $\mathcal{A}=\left\{a_{1}, \ldots, a_{t}\right\}$ be a set $t$ co-prime integers.

- An integer $X$ such that $0 \leq X<A=\prod_{i=1}^{t} a_{i}$ is represented by

$$
[X]_{\mathcal{A}}=\left(x_{1}=X \bmod a_{1}, \ldots, x_{t}=X \bmod a_{t}\right) .
$$

- The Chinese remainder theorem tell us that for op $\in\{+, \times\}$

$[X]_{\mathcal{A}}$ op $[Y]_{\mathcal{A}}=\left(\left[x_{1} \text { op } y_{1}\right]_{a_{1}}, \ldots,\left[x_{t} \text { op } y_{t}\right]_{a_{t}}\right) \Leftrightarrow X$ op $Y \bmod A$ 
Montgomery multiplication in residue number system

- Let $\mathcal{A}=\left\{a_{1}, \ldots, a_{t}\right\}$ be a set $t$ co-prime integers.

- An integer $X$ such that $0 \leq X<A=\prod_{i=1}^{t} a_{i}$ is represented by

$$
[X]_{\mathcal{A}}=\left(x_{1}=X \quad \bmod a_{1}, \ldots, x_{t}=X \quad \bmod a_{t}\right) .
$$

- The Chinese remainder theorem tell us that for op $\in\{+, \times\}$

$$
[X]_{\mathcal{A}} \text { op }[Y]_{\mathcal{A}}=\left(\left[x_{1} \text { op } y_{1}\right]_{a_{1}}, \ldots,\left[x_{t} \text { op } y_{t}\right]_{a_{t}}\right) \Leftrightarrow X \text { op } Y \bmod A
$$

\section{Montgomery Multiplication in RNS}

Require: $X, Y$ in $\mathcal{A} \cup \mathcal{B}$

Ensure: $X Y A^{-1} \bmod N$ in $\mathcal{A} \cup \mathcal{B}$

$1:[Q]_{\mathcal{A}} \leftarrow\left[X Y N^{-1}\right]_{\mathcal{A}}$

3: $[Z]_{\mathcal{B}} \leftarrow\left[(X Y-Q N) A^{-1}\right]_{\mathcal{B}}$

5: return $\left(Z_{\mathcal{A} \cup \mathcal{B}}\right)$ 
Montgomery multiplication in residue number system

- Let $\mathcal{A}=\left\{a_{1}, \ldots, a_{t}\right\}$ be a set $t$ co-prime integers.

- An integer $X$ such that $0 \leq X<A=\prod_{i=1}^{t} a_{i}$ is represented by

$$
[X]_{\mathcal{A}}=\left(x_{1}=X \quad \bmod a_{1}, \ldots, x_{t}=X \quad \bmod a_{t}\right) .
$$

- The Chinese remainder theorem tell us that for op $\in\{+, \times\}$

$$
[X]_{\mathcal{A}} \text { op }[Y]_{\mathcal{A}}=\left(\left[x_{1} \text { op } y_{1}\right]_{a_{1}}, \ldots,\left[x_{t} \text { op } y_{t}\right]_{a_{t}}\right) \Leftrightarrow X \text { op } Y \bmod A
$$

\section{Montgomery Multiplication in RNS}

Require: $X, Y$ in $\mathcal{A} \cup \mathcal{B}$

Ensure: $X Y A^{-1} \bmod N$ in $\mathcal{A} \cup \mathcal{B}$

1: $[Q]_{\mathcal{A}} \leftarrow\left[X Y N^{-1}\right]_{\mathcal{A}}$

2: $[Q]_{\mathcal{B}} \leftarrow B E_{\mathcal{A} \rightarrow \mathcal{B}}\left([Q]_{\mathcal{A}}\right)$

3: $[Z]_{\mathcal{B}} \leftarrow\left[(X Y-Q N) A^{-1}\right]_{\mathcal{B}}$

4: $[Z]_{\mathcal{A}} \leftarrow B E_{\mathcal{B} \rightarrow \mathcal{A}}\left([Z]_{\mathcal{B}}\right)$

5: return $\left(Z_{\mathcal{A} \cup \mathcal{B}}\right)$ 


\section{Outline}

- RSA cryptosystem

- Power analysis

- Montgomery multiplication in RNS

(2) Randomized modular exponentiation in RNS

- Randomized Montgomery multiplication

- Proposed approach

- Level of randomization 


\section{Randomization in RNS (LRA CHES 2004)}

We have

$$
\widetilde{X}_{\text {old }}=\left[X A_{\text {old }}\right]_{\mathcal{A}_{\text {old }} \cup \mathcal{B}_{\text {old }}}
$$

we permute the basis elements $\mathcal{A}_{\text {old }} \cup \mathcal{B}_{\text {old }} \rightarrow \mathcal{A}_{\text {new }} \cup \mathcal{B}_{\text {new }}$

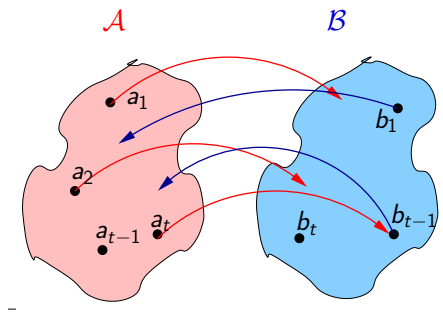

this leads to a new representation of $X$

$$
\widetilde{X}_{\text {new }}=\left[X A_{\text {new }}\right]_{\mathcal{A}_{\text {new }} \cup \mathcal{B}_{\text {new }}}
$$

\section{Cost}

Two Montgomery multiplications :

$X A_{\text {old }} \bmod N \rightarrow X A_{\text {old }} A_{\text {new }} \bmod N \rightarrow X A_{\text {new }} \bmod N$. 


\section{Randomized square-and-multiply-always}

- Input: $N, X \in\left[0, N\left[, E=\left(e_{\ell-1}, \ldots, e_{0}\right)_{2}\right.\right.$ and $\mathcal{M}=\left\{m_{1}, \ldots, m_{2 t}\right\}$.

- Output: $X^{E} \bmod N$

\section{Square-and-mult-always}

$\mathcal{A}, \mathcal{B} \leftarrow$ random split $\mathcal{M}$

$\widetilde{Z} \leftarrow[\widetilde{X}]_{\mathcal{A} \cup \mathcal{B}}$,

$\widetilde{R}_{0} \leftarrow[\widetilde{1}]_{\mathcal{A} \cup \mathcal{B}}, \widetilde{R}_{1} \leftarrow[\widetilde{1}]_{\mathcal{A} \cup \mathcal{B}}$

for $i$ from 0 to $\ell-1$ do

$\widetilde{R}_{e_{i}} \leftarrow \operatorname{MM} \_\operatorname{RNS}\left(\widetilde{R}_{e_{i}}, \widetilde{Z}, \mathcal{A}, \mathcal{B}\right)$

$\widetilde{Z} \leftarrow \operatorname{MM} \_\operatorname{RNS}(\widetilde{Z}, \widetilde{Z}, \mathcal{A}, \mathcal{B})$

end for

return $\widetilde{R}_{1}$ 


\section{Randomized square-and-multiply-always}

- Input: $N, X \in\left[0, N\left[, E=\left(e_{\ell-1}, \ldots, e_{0}\right)_{2}\right.\right.$ and $\mathcal{M}=\left\{m_{1}, \ldots, m_{2 t}\right\}$.

- Output: $X^{E} \bmod N$

\section{Randomized}

Square-and-mult-always

$\mathcal{A}, \mathcal{B} \leftarrow$ random split $\mathcal{M}$

$\widetilde{Z} \leftarrow[\widetilde{X}]_{\mathcal{A} \cup \mathcal{B}}$,

$\widetilde{R}_{0} \leftarrow[\widetilde{1}]_{\mathcal{A} \cup \mathcal{B}}, \widetilde{R}_{1} \leftarrow[\widetilde{1}]_{\mathcal{A} \cup \mathcal{B}}$

for $i$ from 0 to $\ell-1$ do

$\widetilde{R}_{e_{i}} \leftarrow \operatorname{MM}$ RNS $\left(\widetilde{R}_{e_{i}}, \widetilde{Z}, \mathcal{A}, \mathcal{B}\right)$

$\widetilde{Z} \leftarrow \operatorname{MM} \_$RNS $(\widetilde{Z}, \widetilde{Z}, \mathcal{A}, \mathcal{B})$

Randomise $\left(\mathcal{A}_{\text {old }}, \mathcal{B}_{\text {old }}, \mathcal{A}, \mathcal{B}\right)$

$\widetilde{Z} \leftarrow \operatorname{Update}\left(\widetilde{Z}, \mathcal{A}_{\text {old }}, \mathcal{B}_{\text {old }}, \mathcal{A}, \mathcal{B}\right)$

$\widetilde{R}_{0} \leftarrow \operatorname{Update}\left(\widetilde{R}_{0}, \mathcal{A}_{\text {old }}, \mathcal{B}_{\text {old }}, \mathcal{A}, \mathcal{B}\right)$

$\widetilde{R}_{1} \leftarrow \operatorname{Update}\left(\widetilde{R}_{1}, \mathcal{A}_{\text {old }}, \mathcal{B}_{\text {old }}, \mathcal{A}, \mathcal{B}\right)$

\section{end for}

return $\widetilde{R}_{1}$ 


\section{Randomized square-and-multiply-always}

- Input: $N, X \in\left[0, N\left[, E=\left(e_{\ell-1}, \ldots, e_{0}\right)_{2}\right.\right.$ and $\mathcal{M}=\left\{m_{1}, \ldots, m_{2 t}\right\}$.

- Output: $X^{E} \bmod N$

\section{Randomized}

Square-and-mult-always

$\mathcal{A}, \mathcal{B} \leftarrow$ random split $\mathcal{M}$

$\widetilde{Z} \leftarrow[\widetilde{X}]_{\mathcal{A} \cup \mathcal{B}}$,

$\widetilde{R}_{0} \leftarrow\left[\tilde{1}_{\mathcal{A} \cup \mathcal{B}}, \widetilde{R}_{1} \leftarrow[\widetilde{1}]_{\mathcal{A} \cup \mathcal{B}}\right.$

for $i$ from 0 to $\ell-1$ do

$\widetilde{R}_{e_{i}} \leftarrow \operatorname{MM}$ RNS $\left(\widetilde{R}_{e_{i}}, \widetilde{Z}, \mathcal{A}, \mathcal{B}\right)$

$\widetilde{Z} \leftarrow \operatorname{MM} \_$RNS $(\widetilde{Z}, \widetilde{Z}, \mathcal{A}, \mathcal{B})$

Randomise $\left(\mathcal{A}_{\text {old }}, \mathcal{B}_{\text {old }}, \mathcal{A}, \mathcal{B}\right)$

$\widetilde{Z} \leftarrow \operatorname{Update}\left(\widetilde{Z}, \mathcal{A}_{\text {old }}, \mathcal{B}_{\text {old }}, \mathcal{A}, \mathcal{B}\right)$

$\widetilde{R}_{0} \leftarrow \operatorname{Update}\left(\widetilde{R}_{0}, \mathcal{A}_{\text {old }}, \mathcal{B}_{\text {old }}, \mathcal{A}, \mathcal{B}\right)$

$\widetilde{R}_{1} \leftarrow \operatorname{Update}\left(\widetilde{R}_{1}, \mathcal{A}_{\text {old }}, \mathcal{B}_{\text {old }}, \mathcal{A}, \mathcal{B}\right)$

end for

return $\widetilde{R}_{1}$ 
Randomized square-and-multiply-always

- Input: $N, X \in\left[0, N\left[, E=\left(e_{\ell-1}, \ldots, e_{0}\right)_{2}\right.\right.$ and $\mathcal{M}=\left\{m_{1}, \ldots, m_{2 t}\right\}$.

- Output: $X^{E} \bmod N$

\section{Randomized}

Square-and-mult-always

$\mathcal{A}, \mathcal{B} \leftarrow$ random split $\mathcal{M}$

$\widetilde{Z} \leftarrow[\widetilde{X}]_{\mathcal{A} \cup \mathcal{B}}$,

$\widetilde{R}_{0} \leftarrow[\tilde{1}]_{\mathcal{A} \cup \mathcal{B}}, \widetilde{R}_{1} \leftarrow[\widetilde{1}]_{\mathcal{A} \cup \mathcal{B}}$

for $i$ from 0 to $\ell-1$ do

$\widetilde{R}_{e_{i}} \leftarrow \operatorname{MM} \_\operatorname{RNS}\left(\widetilde{R}_{e_{i}}, \widetilde{Z}, \mathcal{A}, \mathcal{B}\right)$

$\widetilde{Z} \leftarrow \operatorname{MM} \_\operatorname{RNS}(\widetilde{Z}, \widetilde{Z}, \mathcal{A}, \mathcal{B})$

Randomise $\left(\mathcal{A}_{\text {old }}, \mathcal{B}_{\text {old }}, \mathcal{A}, \mathcal{B}\right)$

$\widetilde{Z} \leftarrow$ Update $\left(\widetilde{Z}, \mathcal{A}_{\text {old }}, \mathcal{B}_{\text {old }}, \mathcal{A}, \mathcal{B}\right)$

$\widetilde{Z}_{0}<\operatorname{Update}\left(\widetilde{R}_{0}, \mathcal{A}_{\text {old }}, B_{\text {old }}, \mathcal{A}, B\right)$

$\widetilde{R}_{1} \longleftarrow \operatorname{Update}\left(\widetilde{R}_{1}, \mathcal{A}_{\text {old }}, \mathcal{B}_{\text {old }}, \mathcal{A}, \mathcal{B}\right)$

\section{end for}

return $\widetilde{R}_{1}$

\section{Proposed}

$\mathcal{A}, \mathcal{B} \leftarrow$ random split $\mathcal{M}$

$\widetilde{Z} \leftarrow[\widetilde{X}]_{\mathcal{A} \cup \mathcal{B}}$

$\widetilde{R}_{0} \leftarrow[\widetilde{1}]_{\mathcal{A} \cup \mathcal{B}}, \widetilde{R}_{1} \leftarrow[\widetilde{1}]_{\mathcal{A} \cup \mathcal{B}}$

for $i$ from 0 to $\ell-1$ do

$\mathcal{A}_{e_{i}}^{\prime}, \mathcal{B}_{e_{i}}^{\prime} \leftarrow$ random split $\mathcal{M}$

$\widetilde{R}_{e_{i}} \leftarrow \operatorname{MM}$ RNS $\left(\widetilde{R}_{e_{i}}, \widetilde{Z}, \mathcal{A}_{e_{i}}^{\prime}, \mathcal{B}_{e_{i}}^{\prime}\right)$

$\widetilde{Z} \leftarrow \operatorname{MM} \operatorname{RNS}(\widetilde{Z}, \widetilde{Z}, \mathcal{A}, \mathcal{B})$

Randomise $\left(\mathcal{A}_{\text {old }}, \mathcal{B}_{\text {old }}, \mathcal{A}, \mathcal{B}\right)$

$\widetilde{Z} \leftarrow \operatorname{Update}\left(\widetilde{Z}, \mathcal{A}_{\text {old }}, \mathcal{B}_{\text {old }}, \mathcal{A}, \mathcal{B}\right)$

end for

return $\widetilde{R}_{1}$ 
Example

For $E=7=(111)_{2}$ and $\mathcal{M}=\left\{m_{1}, m_{2}, m_{3}, m_{4}\right\}$ 


\section{Example}

For $E=7=(111)_{2}$ and $\mathcal{M}=\left\{m_{1}, m_{2}, m_{3}, m_{4}\right\}$

- Initialization: $\mathcal{A}=\left\{m_{1}, m_{2}\right\}, \mathcal{B}=\left\{m_{3}, m_{4}\right\}$ leads to

$$
\begin{aligned}
R_{1} & =m_{1} m_{2} \bmod N \\
Z & =X m_{1} m_{2} \bmod N
\end{aligned}
$$




\section{Example}

For $E=7=(111)_{2}$ and $\mathcal{M}=\left\{m_{1}, m_{2}, m_{3}, m_{4}\right\}$

- Initialization: $\mathcal{A}=\left\{m_{1}, m_{2}\right\}, \mathcal{B}=\left\{m_{3}, m_{4}\right\}$ leads to

$$
\begin{aligned}
R_{1} & =m_{1} m_{2} \bmod N \\
Z & =X m_{1} m_{2} \bmod N
\end{aligned}
$$

- Loop 1: $\mathcal{A}_{1}=\left\{m_{2}, m_{4}\right\}, \mathcal{B}_{1}=\left\{m_{1}, m_{3}\right\}$ we get

$$
R_{1}=\left(m_{1} m_{2}\right) \times \underbrace{\left(X m_{1} m_{2}\right)}_{Z} \times \underbrace{\left(m_{2}^{-1} m_{4}^{-1}\right)}_{\text {Mont. factor }}=X m_{1}^{2} m_{2} m_{4}^{-1}
$$




\section{Example}

For $E=7=(111)_{2}$ and $\mathcal{M}=\left\{m_{1}, m_{2}, m_{3}, m_{4}\right\}$

- Initialization: $\mathcal{A}=\left\{m_{1}, m_{2}\right\}, \mathcal{B}=\left\{m_{3}, m_{4}\right\}$ leads to

$$
\begin{aligned}
R_{1} & =m_{1} m_{2} \bmod N \\
Z & =X m_{1} m_{2} \bmod N
\end{aligned}
$$

- Loop 1: $\mathcal{A}_{1}=\left\{m_{2}, m_{4}\right\}, \mathcal{B}_{1}=\left\{m_{1}, m_{3}\right\}$ we get

$$
R_{1}=\left(m_{1} m_{2}\right) \times \underbrace{\left(X m_{1} m_{2}\right)}_{Z} \times \underbrace{\left(m_{2}^{-1} m_{4}^{-1}\right)}_{\text {Mont. factor }}=X m_{1}^{2} m_{2} m_{4}^{-1}
$$

$\mathcal{A}=\left\{m_{1}, m_{3}\right\}, \mathcal{B}=\left\{m_{2}, m_{4}\right\}$ leads to

$$
Z=X^{2} m_{1} m_{3}
$$




\section{Example}

For $E=7=(111)_{2}$ and $\mathcal{M}=\left\{m_{1}, m_{2}, m_{3}, m_{4}\right\}$

- Initialization: $\mathcal{A}=\left\{m_{1}, m_{2}\right\}, \mathcal{B}=\left\{m_{3}, m_{4}\right\}$ leads to

$$
\begin{aligned}
R_{1} & =m_{1} m_{2} \quad \bmod N \\
Z & =X m_{1} m_{2} \bmod N
\end{aligned}
$$

- Loop 1: $\mathcal{A}_{1}=\left\{m_{2}, m_{4}\right\}, \mathcal{B}_{1}=\left\{m_{1}, m_{3}\right\}$ we get

$$
R_{1}=\left(m_{1} m_{2}\right) \times \underbrace{\left(X m_{1} m_{2}\right)}_{Z} \times \underbrace{\left(m_{2}^{-1} m_{4}^{-1}\right)}_{\text {Mont. factor }}=X m_{1}^{2} m_{2} m_{4}^{-1}
$$

$\mathcal{A}=\left\{m_{1}, m_{3}\right\}, \mathcal{B}=\left\{m_{2}, m_{4}\right\}$ leads to

$$
Z=X^{2} m_{1} m_{3}
$$

- Loop 2: $\mathcal{A}_{1}=\left\{m_{1}, m_{4}\right\}, \mathcal{B}_{1}=\left\{m_{2}, m_{3}\right\}$ we get

$$
R_{1}=X m_{1}^{2} m_{2} m_{4}^{-1} \times\left(X^{2} m_{1} m_{3}\right) \times\left(m_{1}^{-1} m_{4}^{-1}\right)=X^{3} m_{1}^{2} m_{2} m_{3} m_{4}^{-2}
$$




\section{Example}

For $E=7=(111)_{2}$ and $\mathcal{M}=\left\{m_{1}, m_{2}, m_{3}, m_{4}\right\}$

- Initialization: $\mathcal{A}=\left\{m_{1}, m_{2}\right\}, \mathcal{B}=\left\{m_{3}, m_{4}\right\}$ leads to

$$
\begin{aligned}
R_{1} & =m_{1} m_{2} \quad \bmod N \\
Z & =X m_{1} m_{2} \bmod N
\end{aligned}
$$

- Loop 1: $\mathcal{A}_{1}=\left\{m_{2}, m_{4}\right\}, \mathcal{B}_{1}=\left\{m_{1}, m_{3}\right\}$ we get

$$
R_{1}=\left(m_{1} m_{2}\right) \times \underbrace{\left(X m_{1} m_{2}\right)}_{Z} \times \underbrace{\left(m_{2}^{-1} m_{4}^{-1}\right)}_{\text {Mont. factor }}=X m_{1}^{2} m_{2} m_{4}^{-1}
$$

$\mathcal{A}=\left\{m_{1}, m_{3}\right\}, \mathcal{B}=\left\{m_{2}, m_{4}\right\}$ leads to

$$
Z=X^{2} m_{1} m_{3}
$$

- Loop 2: $\mathcal{A}_{1}=\left\{m_{1}, m_{4}\right\}, \mathcal{B}_{1}=\left\{m_{2}, m_{3}\right\}$ we get

$$
R_{1}=X m_{1}^{2} m_{2} m_{4}^{-1} \times\left(X^{2} m_{1} m_{3}\right) \times\left(m_{1}^{-1} m_{4}^{-1}\right)=X^{3} m_{1}^{2} m_{2} m_{3} m_{4}^{-2}
$$

- Etc. 


\section{Random evolution of the mask}

After $i$ loop iterations we have

$$
\widetilde{R}_{1}^{(i)}=X^{\sum_{j=0}^{i-1} e_{j} 2^{j}} \times \prod_{j=0}^{2 t} m_{j}^{\gamma_{j}^{(i)}} \bmod N
$$

and each $\gamma_{j}^{(i)}$ evolves randomly as

$$
\gamma_{j}^{(i+1)}=\gamma_{j}^{(i)}+\delta_{j}^{(i)} \text { with } \delta_{j}^{(i)} \in\{-1,0,1\} \text { and }\left\{\begin{array}{c}
\mathbb{P}\left(\delta_{j}^{(i)}=1\right)=1 / 8 \\
\mathbb{P}\left(\delta_{j}^{(i)}=-1\right)=1 / 8 \\
\mathbb{P}\left(\delta_{j}^{(i)}=0\right)=3 / 4
\end{array}\right.
$$

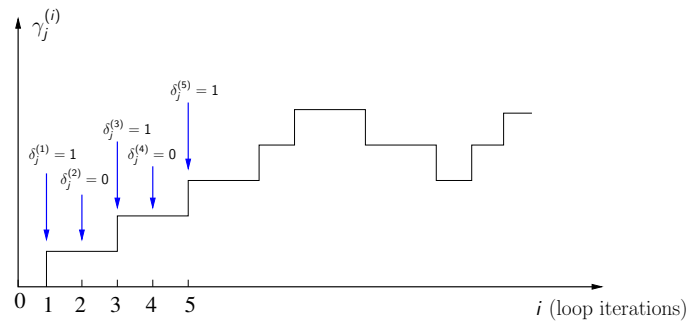




\section{Removing the final mask}

Problem: at the end we have to remove the final mask $\prod_{j=1}^{2 t} m_{j}^{\gamma_{j}^{(\ell)}}$ from

$$
\widetilde{X}=X^{E} \cdot \prod_{j=1}^{2 t} m_{j}^{\gamma_{j}^{(\ell)}} \bmod N
$$

Strategy: we force $\gamma_{j}^{(\ell)}$ to be equal 0 as follows

- During the first half of the iterations each $\gamma_{j}^{(i)}$ evolves freely.

- During the second half we constrain each $\left|\gamma_{j}^{(i)}\right|$ to decrease toward 0.

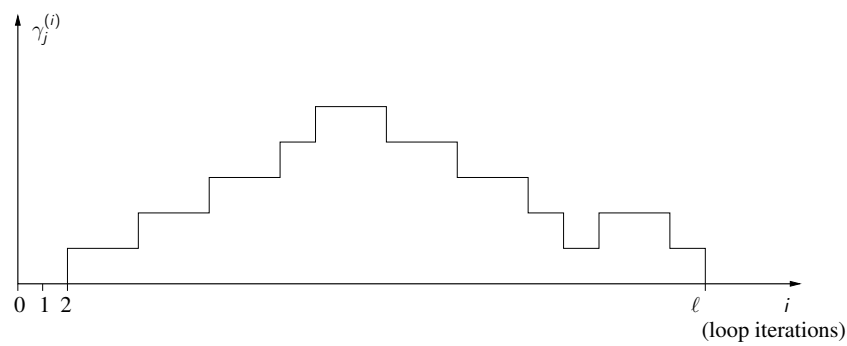




\section{Level of randomization}

- The probabilities of the mask exponents satisfy

$$
\begin{aligned}
& \mathbb{P}\left(\gamma_{j}^{(i)}=d\right)=\sum_{k=d}^{d+\lfloor(i-d) / 2\rfloor}\left(\begin{array}{l}
i \\
k
\end{array}\right)\left(\begin{array}{l}
i-k \\
k-d
\end{array}\right)\left(\frac{1}{8}\right)^{2 k-d}\left(\frac{3}{4}\right)^{i-2 k+d} \\
& \mathbb{P}\left(\Gamma^{(i)}=\Gamma\right) \leq \prod_{j=1}^{t} \mathbb{P}\left(\gamma_{j}^{(i)}=\gamma_{j}\right) \leq \prod_{j=1}^{t} \mathbb{P}\left(\gamma_{j}^{(i)}=0\right)
\end{aligned}
$$




\section{Level of randomization}

- The probabilities of the mask exponents satisfy

$$
\begin{aligned}
& \mathbb{P}\left(\gamma_{j}^{(i)}=d\right)=\sum_{k=d}^{d+\lfloor(i-d) / 2\rfloor}\left(\begin{array}{c}
i \\
k
\end{array}\right)\left(\begin{array}{c}
i-k \\
k-d
\end{array}\right)\left(\frac{1}{8}\right)^{2 k-d}\left(\frac{3}{4}\right)^{i-2 k+d} \\
& \mathbb{P}\left(\Gamma^{(i)}=\Gamma\right) \leq \prod_{j=1}^{t} \mathbb{P}\left(\gamma_{j}^{(i)}=\gamma_{j}\right) \leq \prod_{j=1}^{t} \mathbb{P}\left(\gamma_{j}^{(i)}=0\right)
\end{aligned}
$$

- Comparison: for a 2048-bit RSA modulus and $t=32$ :

- CHES 04:

$\star$ Montgomery-ladder,

$\star$ 4MM_RNS per randomization,

$\star$ all masks are controled.

- Proposed:

$\star$ right-left square-and-multiply-always,

$\star$ 2MM_RNS per randomization

$\star$ the masks for $R_{0}$ and $R_{1}$ are not controled.

\begin{tabular}{|c||c|c|c|c|c|}
\hline Approach & loop 1 & loop 5 & loop 10 & loop 50 & loop 100 \\
\hline \hline CHES 04 & $4.17 \cdot 10^{-38}$ & $4.17 \cdot 10^{-38}$ & $4.17 \cdot 10^{-38}$ & $4.17 \cdot 10^{-38}$ & $4.17 \cdot 10^{-38}$ \\
\hline Proposed & $10^{-8}$ & $5 \cdot 10^{-28}$ & $1.7 \cdot 10^{-38}$ & $2.69 \cdot 10^{-61}$ & $5.75 \cdot 10^{-71}$ \\
\hline
\end{tabular}




\section{Outline}

(1) Cryptography

- RSA cryptosystem

- Power analysis

- Montgomery multiplication in RNS

(2) Randomized modular exponentiation in RNS

- Randomized Montgomery multiplication

- Proposed approach

- Level of randomization

(3) Conclusion 


\section{Conclusion}

Secure embedded implementation of RSA:

- Randomized modular exponentiation

- But leak resistant arithmetic (CHES 04) is costly: 4 MM_RNS per randomization 


\section{Conclusion}

Secure embedded implementation of RSA:

- Randomized modular exponentiation

- But leak resistant arithmetic (CHES 04) is costly: 4 MM_RNS per randomization

We proposed:

- To apply LRA to right-to-left exponentiation.

- Avoid some correction of Montgomery Factor.

- This decreases the computational cost: 2 MM_RNS per randomization.

- Increases the level of randomization after a small number of loop. 


\section{Conclusion}

Secure embedded implementation of RSA:

- Randomized modular exponentiation

- But leak resistant arithmetic (CHES 04) is costly: 4 MM_RNS per randomization

We proposed:

- To apply LRA to right-to-left exponentiation.

- Avoid some correction of Montgomery Factor.

- This decreases the computational cost: 2 MM_RNS per randomization.

- Increases the level of randomization after a small number of loop. Perspectives:

- A better estimation of the level of randomization.

- Is it a good counter-measure against horizontal power analysis ? 
Thank you for your attention! 